\title{
Gamma-ray anisotropies with the Cherenkov Telescope Array
}

\author{
Mattia Fornasa* \\ School of Physics and Astronomy, University of Nottingham, NG7 2RD University Park, \\ Nottingham, United Kingdom \\ E-mail: fornasamegmail.com
}

The Diffuse Gamma-Ray Background (DGRB) is the radiation that remains after the subtraction of the resolved sources and the Galactic foreground from the total gamma-ray emission. The DGRB originates from the cumulative emission of unresolved sources like blazars, star-forming and radio galaxies, but its exact composition remains unknown. Gamma rays produced by Dark Matter (DM) annihilations or decays in Galactic and extragalactic (sub)halos can also contribute, so that the study of the DGRB represents a powerful way to indirectly look for DM. I will summarize how anisotropies in the DGRB can be detected by the Cherenkov Telescope Array (CTA) and show what is the impact of such measurement for the reconstruction of the DGRB nature and of the DM-induced component.

Science with the New Generation of High Energy Gamma-ray experiments, 10th Workshop 04-06 June 2014

Lisbon - Portugal

* Speaker. 


\section{Introduction}

The Diffuse Gamma-Ray Background (DGRB) is the radiation remaining after the resolved sources (both point-like and extended) and the so-called Galactic diffuse foreground (due to interactions of Cosmic Rays with the interstellar medium and interstellar radiation field of the Milky Way) are subtracted from the total gamma-ray emission detected, e.g., from the Fermi-LAT telescope. The most recent measurement of the DGRB energy spectrum is reported in Ref. [1], from the analysis of 50 months of Fermi-LAT data at Galactic latitudes $|b|>20^{\circ}$. The results of Ref. [1] are shown as black data points in Fig. 1 and they extend the previous observation of the DGRB energy spectrum down to $100 \mathrm{MeV}$ and up to $820 \mathrm{GeV}$. The emission is compatible with a power-law spectrum with a spectral index of $2.31 \pm 0.02$ and an exponential cut-off starting from $279 \pm 52$ $\mathrm{GeV}$. The largest systematic uncertainty in the determination of the DGRB comes from our imperfect knowledge of the diffuse Galactic foreground and it ranges from a factor of $\sim 15 \%$ to $30 \%$ depending on the energy range.

The DGRB is interpreted as the cumulative emission produced by all those sources that are not bright enough to be detected individually. Unresolved blazars, misaligned Active Galactic Nuclei (MAGNs), star-forming galaxies (SFGs) and millisecond pulsars (MSPs) are guaranteed components to the DGRB. The contribution of MSPs is subdominant [2], while that of the other three classes is summarized by the coloured bands in Fig. 1. Note that blazars have been divided into Flat-Spectrum Radio Quasars (FSRQs) and BL Lacs. The figure also shows that these 4 components (FSRQs, BL Lacs, MAGNs and SFGs) are enough to reproduce the energy spectrum of the DGRB, as measured by Fermi-LAT in Ref. [1]. This automatically constrains the contribution of any other gamma-ray emitters as, e.g., galaxy clusters or Type Ia supernovae. It also provides constraints to more exotic gamma-ray emissions, as the one expected from the annihilations or decays of Dark Matter (DM) particles [3, 4].

The intensity $I_{\mathrm{DM}}(\mathbf{n})$ of the DM-induced emission from direction $\mathbf{n}$ in the sky depends on the properties of the DM particle, e.g. its mass $m_{\chi}$, annihilation cross section $\left\langle\sigma_{\mathrm{ann}} v\right\rangle$ or decay lifetime $\tau$. Here we focus on a class of DM candidates called Weakly Interacting Massive Particles (WIMPs), characterized by a GeV-TeV mass and by weak-scale interactions. Also, the DMinduced gamma-ray flux rests on the abundance and the properties of DM structures, which can be inferred from $N$-body simulations.

The measured DGRB energy spectrum can be used to constrain the intensity of the DMinduced emission and, thus, to derive upper (lower) limits on the annihilation cross section (decay lifetime), as a function of the DM mass. Below the TeV scale, the so-called "sensitivity reach" limits derived in Ref. [5] from the DGRB energy spectrum in Ref. [1] represent the strongest constraint currently available on $\left\langle\sigma_{\mathrm{ann}} v\right\rangle$. Similarly, in the case of decaying DM, the lower limits on $\tau$ obtained in Ref. [6] from the DGRB measurement in Ref. [7] are the most constraining, up to 20 $\mathrm{TeV}$.

\section{Anisotropies in the Diffuse Gamma-Ray Background}

Before the Fermi-LAT, the energy spectrum was the only source of information available on the DGRB. However, in 2012, Fermi-LAT measured, for the first time, also the angular power 


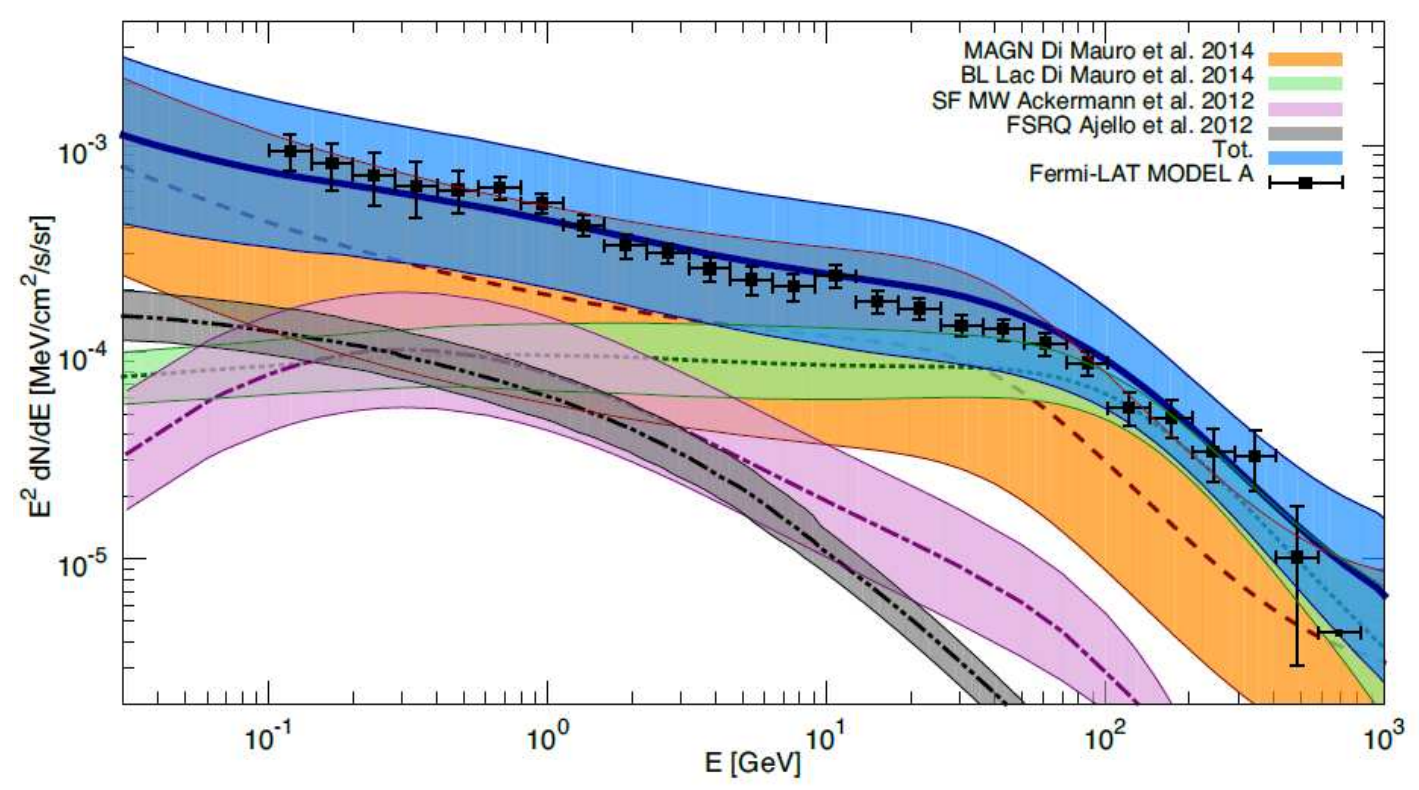

Figure 1: The black data points represent the most recent measurement of the energy spectrum of the DGRB by Fermi-LAT [1]. Model A from Ref. [1] is adopted for the diffuse Galactic foreground. The coloured lines indicate the expected gamma-ray emission from unresolved sources, for 4 different wellestablished astrophysical populations: BL Lacs (short-dashed green), FSRQs (double-dotted-dashed gray), SFGs (dotted-dashed purple) and MAGNs (long-dashed orange). Each colour bar represents the expected uncertainty on the emission of each population. The sum of these contributions is denoted by the solid blue line and the blue uncertainty band. Estimates are taken from Ref. [8] (for BL Lacs), Ref. [9] (for FSRQs), Ref. [10] (for MAGNs) and Ref. [11] (for SFGs). Taken from Ref. [12].

spectrum (APS) of anisotropies in the DGRB [13]. The emission exhibits a Poissonian APS in the angular multipole range between $\ell=155$ and 504, which a significance as large as $7.2 \sigma$ between 1.99 and $5.0 \mathrm{GeV}$. The measured APS between 1.0 and $2.0 \mathrm{GeV}$ can be seen in the left panel of Fig. 2. It is possible to predict how much the unresolved astrophysical sources mentioned above can contribute to the DGRB APS [14]. Results are summarized in the right panel of Fig. 2, showing that a particular class of blazars (called high-synchrotron-peak BL Lacs) can explained the whole APS signal from Ref. [13].

\section{Anisotropies induced by Dark Matter}

As done for the DGRB energy spectrum, it is possible to use the Fermi-LAT measurement of the APS to constrain a possible DM component to the DGRB. The APS $C_{\ell}^{\mathrm{DM}}$ of the DM-induced gamma-ray emission $I_{\mathrm{DM}}(\mathbf{n})$ can be computed by decomposing $I_{\mathrm{DM}}(\mathbf{n})$ in spherical harmonics. Analytical estimations are derived in Ref. [4]. Alternatively, one can employ the results of $\mathrm{N}$ body simulations to build a simulated sky-map of $I_{\mathrm{DM}}(\mathbf{n})$ and, then, compute $C_{\ell}^{\mathrm{DM}}$ by means of the HEALPix package [3]. Both procedure are affected by our lack on knowledge on the abudance and properties of DM structures below the mass resolution of $N$-body simulations. This represents the largest source of uncertainty in the estimation of $C_{\ell}^{\mathrm{DM}}$, which can be as large as 2 orders of 

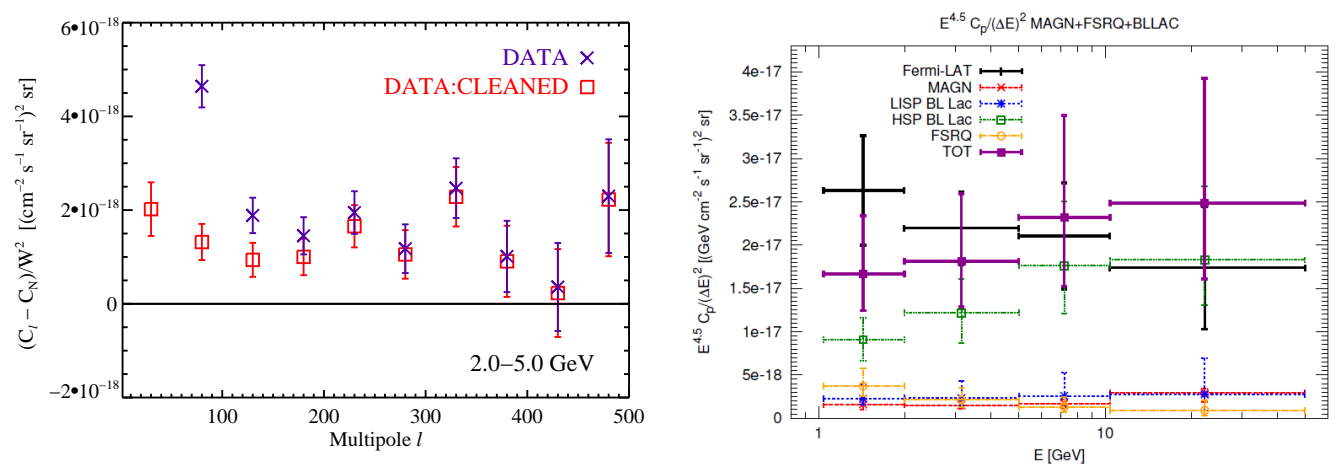

Figure 2: Left: Estimator of the APS of anisotropies considered in Ref. [13] as a function of the angular multipole $\ell$. The purple crosses (red squares) indicate the APS from the Fermi-LAT without (with) subtracting a model for the diffuse Galactic foreground. The signal region in Ref. [13] is defined between $\ell=155$ and 504. Taken from Ref. [13]. Right: Poissonian APS $C_{P}$ (as a function of the energy) expected from MAGNs (long-dashed red), low-synchrotron-peak and intermediate-synchrotron-peak BL Lacs blazars (short-dashed blue), high-synchrotron peak BL Lacs blazars (dotted green), Flat-Spectrum Radio Quasars (dot-dashed yellow) and the sum of the above (solid purple). The black solid data points indicate the measurement from Ref. [13]. Taken from Ref.[14].

magnitude [3]. However, even in the most optimistic (and possibly unrealistic scenario), DMinduced emission does not contribute significantly to the APS detected by the Fermi-LAT and, thus, the upper (lower) limits that can be derived on $\left\langle\sigma_{\mathrm{ann}} v\right\rangle(\tau)$ are not as constraining as those discussed in the previous section.

\section{Measuring the anisotropy angular power spectrum with CTA}

Ref. [15] determines, for the first time, the sensitivity of the Cherenkov Telescope Array (CTA) to the detection of the anisotropy APS and it quantifies how such an observable could help in the reconstruction of the DGRB composition.

Some of the main results of Ref. [15] are summarized in Fig. 3. The different coloured line in the left panel indicate the APS computed by applying HEALPix on simulated all-sky maps of the gamma-ray sky characterized by a APS proportional to $\ell^{-0.5} /(\ell+1)$ (dashed lines). One single field-of-view (FOV) of $2.5^{\circ}$ is considered for the darker shades of colour, while 10 different FOVs are simulated for the lighter colours. Red, green and blue lines correspond to different number of simulated events, corresponding to different experimental exposures. The measured APS is affected by the size of the FOV at low multipoles, so that the signal region starts around $\ell=80$. At large $\ell$, the APS is dominated by the Poissonian term, due to finite statistics (dashed black lines). For the largest number of events (blue lines) there is a multipole range (between approximately $\ell=100$ and 500) where CTA can reproduce the nominal $C_{\ell}$. This multipole window reaches $\ell=2000$ if 10 FOVs are used (light blue line). However it reduces in size for $10^{5}$ simulated events (green bands) and pratically disappears for an exposure corresponding to 1000 events (red bands).

A second, more realistic, test is performed in Ref. [15]. The authors generates two sets of maps in which the total number of "signal" events is chosen to reproduce the total integrated DGRB flux 

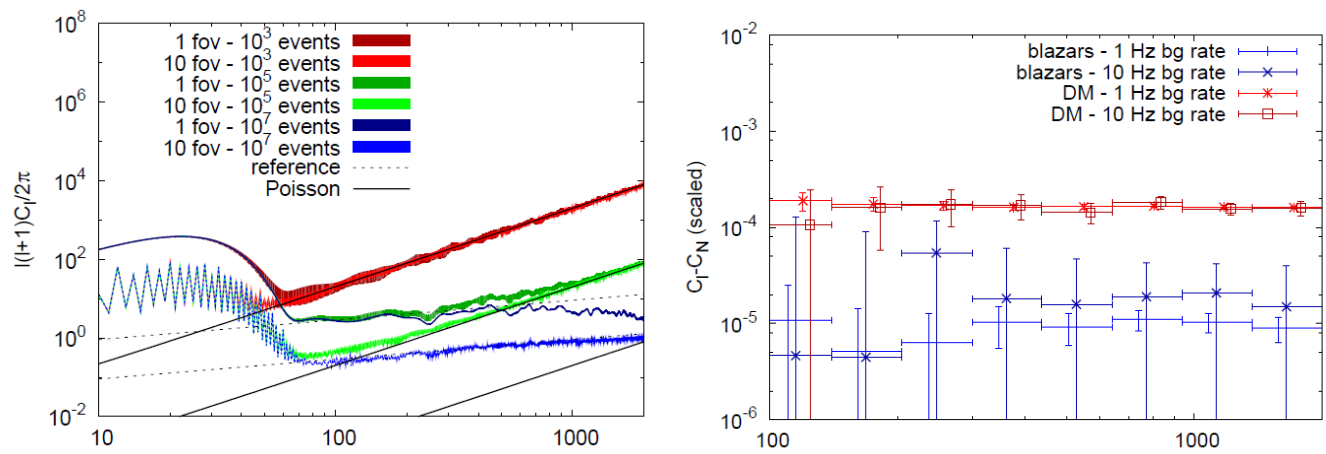

Figure 3: Left: Reconstructed APS for an input APS proportional to $\ell^{-0.5} /(\ell+1)$, for 1000 (red), $10^{5}$ (green), and $10^{7}$ (blue) signal-only events, distributed in a single FOV (dark shades) or in ten different FOVs (light shades), respectively. Note that with the definition of the normalization of the maps adopted in Ref. [15], the Poissonian noise (depicted by the dashed black lines) depends only on the number of events and it is the same in the single or multiple FOV cases. For comparison, the thin dotted lines show the input APS for the two $10^{7}$-event cases. A Gaussian PSF with a width of $0.05^{\circ}$ is assumed. Right: Comparison between the measured APS for a pure astrophysical case with $C_{\ell}=10^{-5}$ (blue and purple data points) and a case with $40 \%$ of the total radiation originating from self-annihilating DM with $C_{\ell}=10^{-3}$ (red and brown data points). An observation with a CTA-like telescope system of $1000 \mathrm{~h}$ on a single FOV is considered. $E_{\mathrm{th}}$ is fixed to $300 \mathrm{GeV}$. A background rate of $1 \mathrm{~Hz}$ is assumed for the blue and red points, while $10 \mathrm{~Hz}$ is considered for the purple and brown ones. The photon noise APS is already subtracted to the raw $C_{\ell}$ provided by HEALPix. Taken from Ref. [15].

reported in Ref. [7], above an energy threshold $E_{\text {thr }}$ and for a certain exposure $\Delta T$. A number of background events associated with that exposure is also simulated. In the first set of maps the DGRB is assumed to be made entirely of unresolved astrophysical sources and the simulated signal events are distributed in order to reproduce a Poissonian APS of $C_{P}^{\text {astro }}=10^{-5}$ [13]. For the second set of maps, a certain fraction $x$ of the signal events are assumed to come from DM annihilations and distributed in order to have a $C_{P}^{\mathrm{DM}}=10^{-3}[16]$. Different values of the DM fraction $x$ are considered and, for each value of $x$, the APS of the "DM+astrophysics" map is compared with the APS of the maps obtained without the DM component. In this way it is possible to determine the smallest fraction of DM for which the "DM+astrophysics" case can be distinguished, within the estimated experimental errors, from the "astrophysics-only" scenario. For an energy threshold of $E_{\mathrm{thr}}=300 \mathrm{GeV}$ and an exposure of $\Delta T=1000$ hours, a CTA measurement of the APS using only 1 FOV can provide a detection of a DM component to the DGRB if such a component is larger than $15 \%$ of the DGRB intensity in Ref. [7] (for a background rate of $1 \mathrm{~Hz}$ ). This can be seen in the right panel of Fig. 3, that shows the estimated APS (after the subtraction of the photon noise). Blue and purple data points correspond to the "astrophysics-only" case while red and brown also contain a $40 \%$ of DM. Blue and red points are for a background rate of $1 \mathrm{~Hz}$ and purple and brown ones for a more pessimistic case of $10 \mathrm{~Hz}$. As it can be seen the red (brown) data are stastically different compared to the blue (purple) ones.

An exposure of 1000 hours is probably unrealistically large, however Ref. [15] proved that splitting the observation into 10 identical FOVs (i.e. having $10 \mathrm{FOVs}$ with a $\Delta T=100$ hours) 
increases the minimal fraction of DM that can be detected only by a factor 2 . The moderate increase is compensated by the fact that the observation pattern is now more realistic.

\section{Conclusions}

Measuring gamma-ray anisotropies with CTA is not an unrealstic goal. Such a measurement would extend the current observation by the Fermi-LAT to the energies larger than $50 \mathrm{GeV}$. It can potentially provide important information on the composition of the DGRB and, in particular, single out a DM component to the DGRB, even in a scenario in which DM is responsible only for $15 \%$ of the DGRB from Ref. [7] above $300 \mathrm{GeV}$.

\section{References}

[1] M. Ackermann et al. [The Fermi LAT Collaboration], arXiv:1410.3696 [astro-ph.HE].

[2] F. Calore, M. Di Mauro, F. Donato and F. Donato, Astrophys. J. 796 (2014) 1 [arXiv:1406.2706 [astro-ph.HE]].

[3] M. Fornasa, J. Zavala, M. A. Sanchez-Conde, J. M. Siegal-Gaskins, T. Delahaye, F. Prada, M. Vogelsberger and F. Zandanel et al., Mon. Not. Roy. Astron. Soc. 1529 (2013) [arXiv:1207.0502 [astro-ph.HE]].

[4] S. i. Ando and E. Komatsu, Phys. Rev. D 87 (2013) 12, 123539 [arXiv:1301.5901 [astro-ph.CO]].

[5] M. Ackermann et al. [ The Fermi LAT Collaboration], arXiv:1501.05464 [astro-ph.CO].

[6] M. Cirelli, E. Moulin, P. Panci, P. D. Serpico and A. Viana, Phys. Rev. D 86 (2012) 083506 [arXiv:1205.5283 [astro-ph.CO]].

[7] A. A. Abdo et al. [Fermi-LAT Collaboration], Phys. Rev. Lett. 104 (2010) 101101 [arXiv:1002.3603 [astro-ph.HE]].

[8] M. Di Mauro, F. Donato, G. Lamanna, D. A. Sanchez and P. D. Serpico, Astrophys. J. 786 (2014) 129 [arXiv:1311.5708 [astro-ph.HE]].

[9] M. Ajello, M. S. Shaw, R. W. Romani, C. D. Dermer, L. Costamante, O. G. King, W. Max-Moerbeck and A. Readhead et al., Astrophys. J. 751 (2012) 108 [arXiv:1110.3787 [astro-ph.CO]].

[10] M. Di Mauro, F. Calore, F. Donato, M. Ajello and L. Latronico, Astrophys. J. 780 (2014) 161 [arXiv:1304.0908 [astro-ph.HE]].

[11] I. Tamborra, S. Ando and K. Murase, JCAP 1409 (2014) 09, 043 [arXiv:1404.1189 [astro-ph.HE]].

[12] M. Di Mauro and F. Donato, arXiv:1501.05316 [astro-ph.HE].

[13] M. Ackermann et al. [Fermi LAT Collaboration], Phys. Rev. D 85 (2012) 083007 [arXiv:1202.2856 [astro-ph.HE]].

[14] M. Di Mauro, A. Cuoco, F. Donato and J. M. Siegal-Gaskins, JCAP 1411 (2014) 11, 021 [arXiv:1407.3275 [astro-ph.HE]].

[15] J. Ripken, A. Cuoco, H. S. Zechlin, J. Conrad and D. Horns, JCAP 1401 (2014) 01, 049 [arXiv:1211.6922 [astro-ph.HE]].

[16] J. M. Siegal-Gaskins, JCAP 0810 (2008) 040 [arXiv:0807.1328 [astro-ph]]. 